\title{
Thermopower enhancement by encapsulating cerium in clathrate cages
}

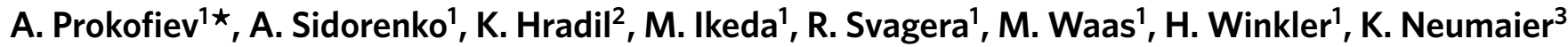 \\ and S. Paschen ${ }^{1 \star}$
}

\begin{abstract}
The increasing worldwide energy consumption calls for the design of more efficient energy systems. Thermoelectrics could be used to convert waste heat back to useful electric energy if only more efficient materials were available. The ideal thermoelectric material combines high electrical conductivity and thermopower with low thermal conductivity. In this regard, the intermetallic type-I clathrates show promise with their exceedingly low lattice thermal conductivities'. Here we report the successful incorporation of cerium as a guest atom into the clathrate crystal structure. In many simpler intermetallic compounds, this rare earth element is known to lead, through the Kondo interaction, to strong correlation phenomena including the occurrence of giant thermopowers at low temperatures ${ }^{2}$. Indeed, we observe a $50 \%$ enhancement of the thermopower compared with a rareearth-free reference material. Importantly, this enhancement occurs at high temperatures and we suggest that a rattlingenhanced Kondo interaction ${ }^{3}$ underlies this effect.
\end{abstract}

Type-I clathrates are guest-host systems with the general composition $\mathrm{G}_{8} \mathrm{H}_{46}$. The guest atoms $\mathrm{G}$ are situated in polyhedral face-sharing and space-filling cages formed by the tetrahedrally bonded host atoms H (Fig. 1a). Exceptionally low lattice thermal conductivities are an intrinsic property of these materials as they are observed even in pure single-crystalline specimens ${ }^{1}$. They have been attributed to reduced phonon group velocities resulting from the interaction of acoustic and rattling modes ${ }^{4,5}$. The charge carriers are essentially unaffected by these lattice anomalies. Thus, type-I clathrates seem to be a realization of the phonon glass-electron crystal concept ${ }^{6}$. With this in mind, many groups worldwide have synthesized and investigated a large number of type-I clathrates with various compositions over the past decades. As a result a significant increase has been achieved in the thermoelectric figureof-merit $Z T=S^{2} \sigma / \kappa \cdot T$, where $T$ is the absolute temperature, $S$ is the thermopower, $\sigma$ is the electrical conductivity and $\kappa$ is the thermal conductivity. The highest $Z T$ values reported are 1.63 at $1,100 \mathrm{~K}$ for n-type $\mathrm{Ba}_{8} \mathrm{Ga}_{16} \mathrm{Ge}_{30}$ (ref. 7) and 1.1 at $900 \mathrm{~K}$ for p-type $\mathrm{Ba}_{8} \mathrm{Ga}_{16} \mathrm{Ge}_{30}$ (ref. 8).

Record high power factors $S^{2} \sigma$ have, however, been found in a very different class of materials-in strongly correlated rare earth or transition metal compounds ${ }^{2,9-11}$. The giant thermopower values observed in these systems can be traced back to an enhanced quasiparticle density of states near the Fermi level that results from the Kondo interaction of the local $4 f$ states with the conduction electrons ${ }^{12,13}$.

Thus, the incorporation of adequate rare earth elements into type-I clathrates seems a promising ${ }^{2}$ yet so far unachieved $^{14-18}$ route to superior thermoelectric materials. In particular, it has not been possible to incorporate any sizable amount of $\mathrm{Ce}$ - the rare earth element with the strongest tendency to hybridize with conduction electrons-into a clathrate phase ${ }^{14-16}$. Here we report on the first successful synthesis of a Ce-containing clathrate and on the finding that its thermopower is sizably enhanced with respect to a rare-earth-free reference material.

In our search for a synthesis route to a Ce-containing clathrate, we were guided by the Zintl-Klemm concept ${ }^{19}$ that predicts that polar intermetallic compounds are stable if all atoms reach filled electron shells. In type-I clathrates one assumes that the host atoms are covalently bound to each other and the guest atoms are ionically bound to the host. For the usual case of electropositive guest atoms this is realized by a transfer of the guest atom's valence electrons to the host, which thus becomes a polyanion. An example is the clathrate $\left(\mathrm{M}^{+2}\right)_{8}\left(\mathrm{III}^{-1}\right)_{16}\left(\mathrm{IV}^{0}\right)_{30}$, where the 2-valent metal atoms $\mathrm{M}$ are the guest atoms and the group III and group IV elements form the framework. The superscripts represent the formal charges of the atoms: the valence electrons of $\mathrm{M}$ are donated to the 3-valent elements III to establish the fourth framework bond. With all valence electrons used up an electrical insulator is expected. Real clathrates are semiconductors or poor metals; this is attributed to slight deviations from the above oversimplified situation.

A particularly broad composition range around the ideal Zintl composition was found in the transition metal clathrate $\mathrm{Ba}_{8} \mathrm{Au}_{x} \mathrm{Si}_{46-x}$ (BAS), which forms in a wide $\mathrm{Au}$ concentration range $2.2 \leq x \leq 6$ (ref. 20) including the ideal Zintl composition $\left(\mathrm{Ba}^{+2}\right)_{8}\left(\mathrm{Au}^{-3}\right)_{5.3}\left(\mathrm{Si}^{0}\right)_{40 . \dot{6}}$ for monovalent Au. This points to an exceptional robustness of this phase, which is why we have chosen it as the starting material for our study.

The substitution of two-valent Ba by three-valent Ce must be accompanied by an increase of the acceptor capacitance of the framework and thus by an enhanced Au content. At $x=6$, the Ce content for charge balance corresponds to the clathrate $\left(\mathrm{Ba}^{+2}\right)_{6}\left(\mathrm{Ce}^{+3}\right)_{2}\left(\mathrm{Au}^{-3}\right)_{6}\left(\mathrm{Si}^{0}\right)_{40}$ with two Ce atoms per formula unit. This ideal Zintl composition was the starting point for our synthesis (Methods), which finally yielded single-crystalline clathrate specimens of the composition $\mathrm{Ba}_{6.91 \pm 0.17} \mathrm{Ce}_{1.06 \pm 0.12} \mathrm{Au}_{5.56 \pm 0.25} \mathrm{Si}_{40.47 \pm 0.43}$ (Ce-BAS, Fig. 1 and Methods). The presence of the sizable amount of about $1 \mathrm{Ce}$ atom per formula unit in the clathrate phase is unambiguously proved by our energy-dispersive X-ray spectroscopy (EDX) and wavelength-dispersive X-ray spectroscopy (WDX) analyses, together with the fact that no foreign phase is present (Fig. 1b,c), even on the nanometre scale (Fig. 1d). It is also in line with the sizable reduction of the lattice parameter, evidenced by the refinement of powder X-ray diffraction (XRD)

\footnotetext{
${ }^{1}$ Institute of Solid State Physics, Vienna University of Technology, Wiedner Hauptstraße 8-10, 1040 Vienna, Austria, ${ }^{2}$ X-Ray Center, Vienna University of Technology, Getreidemarkt 9, 1060 Vienna, Austria, ${ }^{3}$ Walther-Meißner-Institute for Low Temperature Research, Walther-Meißner-Straße 8, 85748 Garching, Germany. ^e-mail:prokofiev@ifp.tuwien.ac.at; paschen@ifp.tuwien.ac.at
} 


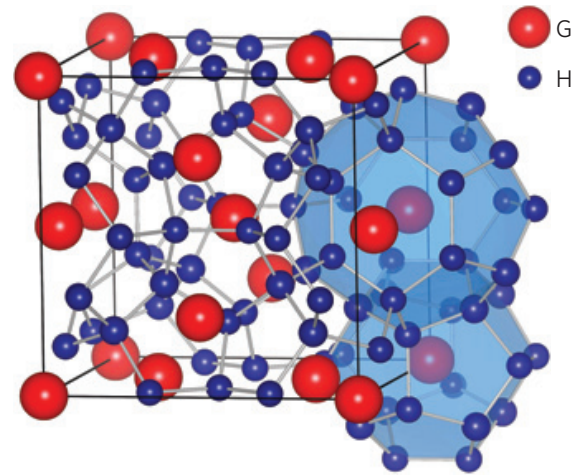

c

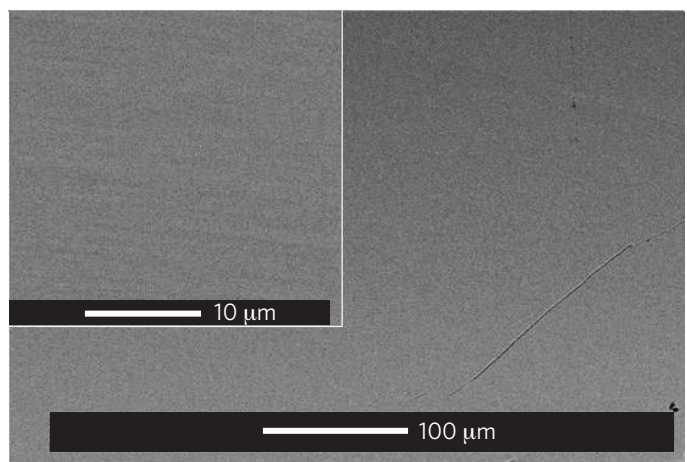

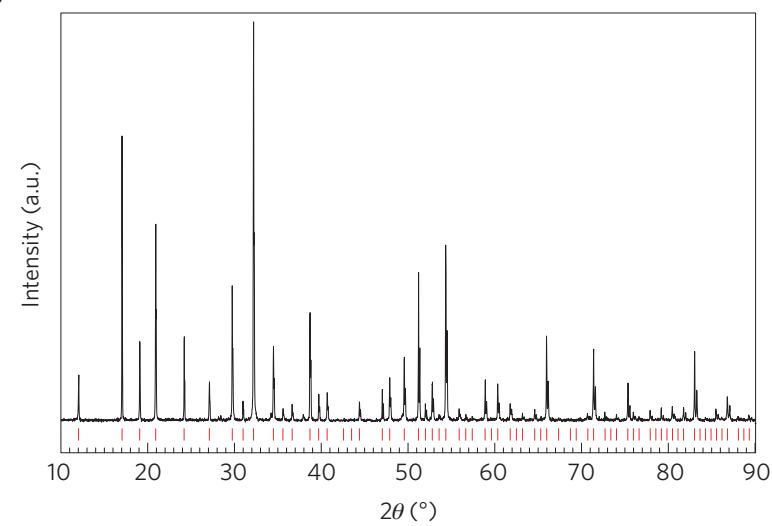

d

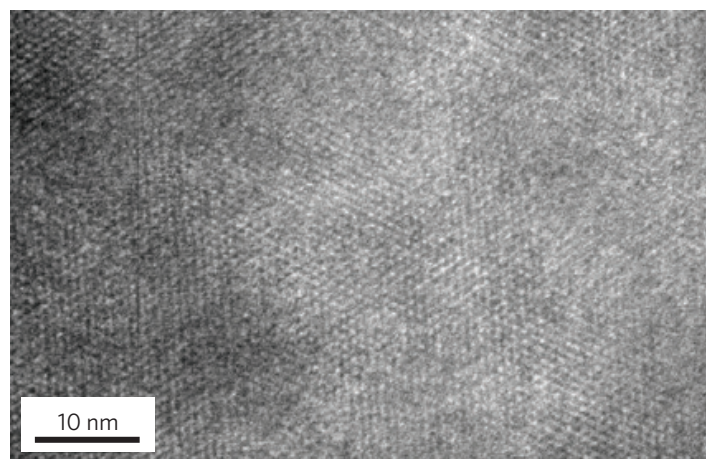

Figure 1 | Structure and quality characterization of the clathrate Ce-BAS. a, Schematic representation of the crystal structure of type-I clathrates. The two different cages are sketched. The guest atoms ( $G$, red) sit at the $2 a(6 c)$ site with cubic (tetragonal) point symmetry in the smaller (larger) cage made up of host atoms ( $\mathrm{H}$, blue). b. The powder XRD pattern (top) can be fully indexed with the type-I clathrate structure (bottom). c, Scanning electron microscopy images at two different magnifications reveal that no micro-inclusions are present. $\mathbf{d}$, The representative transmission electron microscopy image rules out inhomogeneities on the nanometre scale.

data sets (Methods), compared with BAS samples with similar Au content. We also prepared a phase-pure La-containing clathrate $\left(\mathrm{Ba}_{6.99 \pm 0.17} \mathrm{La}_{1.23 \pm 0.12} \mathrm{Au}_{5.91 \pm 0.25} \mathrm{Si}_{39.87 \pm 0.43}\right.$, La-BAS, Methods) as a $4 f$-free reference compound. Structural refinements using singlecrystal XRD data sets (Supplementary Information) reveal that both Ce and La occupy the $2 a$ site within the smaller cage (Fig. 1a).

Having firmly established the presence of $\mathrm{Ce}$ (and La) in our single-phased clathrate samples by analytical and structural investigations we now turn to their physical properties. The electrical resistivity $\rho(T)$ of La-BAS decreases with decreasing temperature, behaviour typical for metals. In contrast, $\rho$ of Ce-BAS increases on cooling (Fig. 2a). This semiconductor-like characteristic is unexpected, as discussed below.

The linear response Hall coefficient $R_{\mathrm{H}}(T)$ is negative for Ce-BAS and positive for La-BAS, in agreement with the Zintl count based on the measured compositions. The absolute value of $R_{\mathrm{H}}$ is distinctly smaller for Ce-BAS than for La-BAS (Fig. 2b), suggesting that $\mathrm{Ce}-\mathrm{BAS}$ has a higher charge carrier concentration and should thus be more metallic than La-BAS. The temperature dependence of $\left|R_{\mathrm{H}}\right|(T)$ is similar for both compounds. It is typical of heavily doped semiconductors. In particular, no hint for an anomalous contribution to the Hall effect ${ }^{21}$ of Ce-BAS can be discerned. Thus, the enhanced resistivity of Ce-BAS is not a charge carrier concentration effect but is instead due to a reduced Hall mobility $\mu_{\mathrm{H}}=\left|R_{\mathrm{H}}\right| / \rho$ (Fig. 2b, inset).

A comparison with published mobility data for a series of polycrystalline BAS samples ${ }^{20}$ reveals that $\mu_{\mathrm{H}}$ of our La-BAS single crystal is significantly enhanced with respect to the p-type BAS sample closest in carrier concentration. This is most naturally attributed to reduced scattering in a single crystal due to the absence of grain boundaries, an effect that was also seen in $\mathrm{Ba}-\mathrm{Ni}-\mathrm{Ge}$ clathrates $^{22}$. In contrast, the mobility of our singlecrystalline Ce-BAS sample is significantly lower than that of the polycrystalline n-type BAS sample of similar carrier concentration. Thus, an additional scattering process must be evoked to explain the low mobility of Ce-BAS.

The most obvious mechanism for a metal containing Ce ions is Kondo scattering. In heavy-fermion metals, incoherent Kondo scattering above the single-ion Kondo temperature $T_{\mathrm{K}}$ leads to a resistivity contribution proportional to $-\ln T$. Owing to the temperature-dependent charge carrier concentration of Ce-BAS, this dependence can be expected to hold only approximately, in agreement with our results (Fig. $2 \mathrm{a}$ ). Below $T_{\mathrm{K}}$, heavy-fermion metals show a pronounced decrease of the resistivity, which is due to the onset of coherence in a Kondo lattice. The absence of this decrease in Ce-BAS is explained as follows. The multiplicity of the $2 a$ site, at which the Ce atoms are situated, is 2. As Ce-BAS contains only $1.06 \mathrm{Ce}$ ions per formula unit it does not occupy this site fully. The $0.94 \mathrm{Ba}$ ions on this site act as Kondo holes ${ }^{23}$ on the lattice, which will inhibit the formation of a coherent state. Magnetic susceptibility and specific heat measurements presented below support the importance of Kondo phenomena in Ce-BAS.

Next, we discuss the thermoelectric properties. The thermopower $S(T)$ is the quantity that is at the very heart of the phenomenon of thermoelectricity. It is negative (positive) for Ce-BAS (La-BAS, Fig. 2c), in agreement with the Hall effect results presented above. Extrema of $-180 \mu \mathrm{V} \mathrm{K}^{-1}\left(300 \mu \mathrm{V} \mathrm{K}^{-1}\right)$ are reached at $480 \mathrm{~K}(375 \mathrm{~K})$ for Ce-BAS (La-BAS). These values exceed those of all previously studied BAS clathrates ${ }^{24,25}$. In the next paragraph we will show that, even though La-BAS reaches higher $S$ values, it is in 


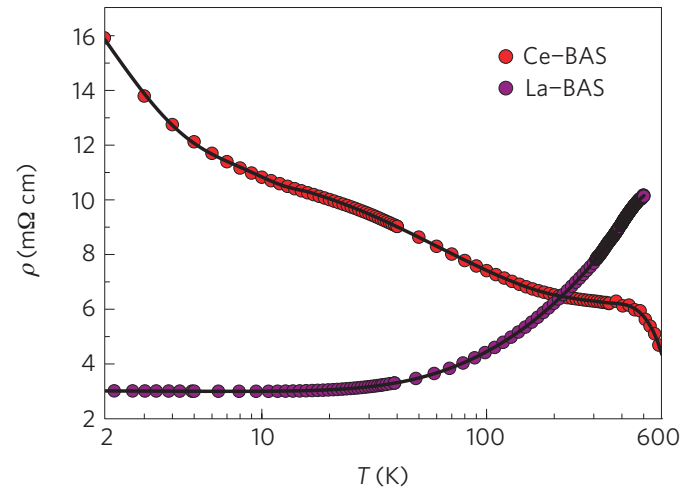

c

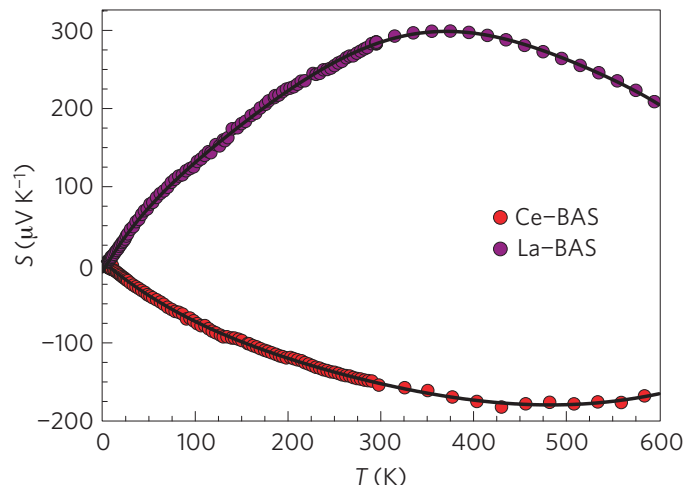

b

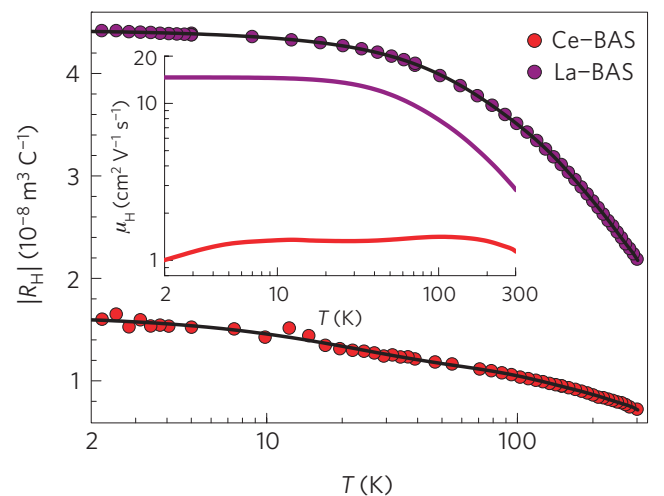

d

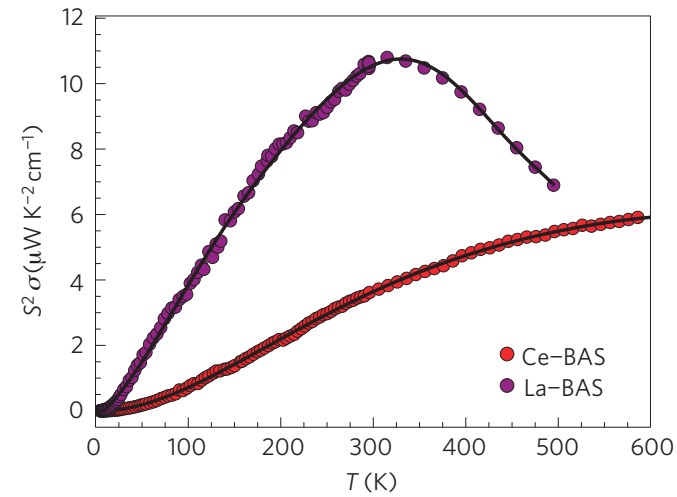

e

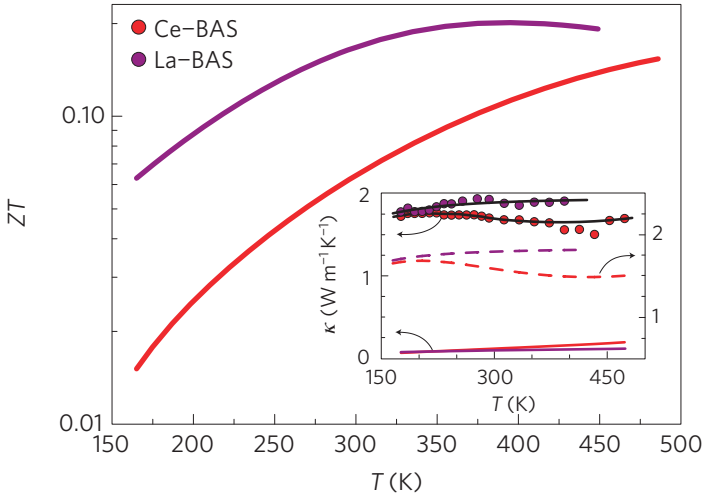

Figure 2 | Transport properties of Ce-BAS and La-BAS. a-e, Temperature dependencies of the electrical resistivity (a), the absolute value of the Hall coefficient (b), the Hall mobility (b, inset), the thermopower (c), the power factor (d), the dimensionless thermoelectric figure of merit (e) and the thermal conductivity $\kappa$ (symbols, left axis), the electronic part $\kappa_{\mathrm{e}}$ estimated using the Wiedemann-Franz law from $\rho(T)$ (solid lines, left axis), and the lattice thermal conductivity $\kappa_{l}=\kappa-\kappa_{\mathrm{e}}$ (dashed lines, right axis) (e, inset). The black lines are guides to the eye. They were used to calculate $\mu_{H}$ and $Z T$.

fact Ce-BAS that shows an anomalous thermopower enhancement. The highest power factors $S^{2} \sigma$ reached in the temperature range $2-600 \mathrm{~K}$ are approximately $11 \mu \mathrm{W} \mathrm{K} \mathrm{K}^{-2} \mathrm{~cm}^{-1}$ for La-BAS at $350 \mathrm{~K}$ and $6 \mu \mathrm{W} \mathrm{K} \mathrm{K}^{-2} \mathrm{~cm}^{-1}$ for Ce-BAS at $600 \mathrm{~K}$, with a tendency to further enhancement at higher temperatures (Fig. 2d). The thermal conductivities $\kappa(T)$ of Ce-BAS and La-BAS (Fig. 2e, inset) are similar to the ones found in the BAS series ${ }^{24}$. As $\kappa(T)$ is dominated by the lattice contribution $\kappa_{1}$ this shows that the rattling modes of Ce-BAS and La-BAS are just as effective in producing a low lattice thermal conductivity as in BAS. For n-type Ce-BAS, the maximum $Z T$ value of about 0.15 at $480 \mathrm{~K}\left(0.19\right.$ at $600 \mathrm{~K}$ if a constant $\kappa_{1}$ above $480 \mathrm{~K}$ is assumed $)$ is $30 \%(100 \%$ at $600 \mathrm{~K})$ higher than that of the best $\mathrm{n}$-type BAS material at the same temperature. For p-type La-BAS, ZT is 0.2 at $400 \mathrm{~K}$, which is $35 \%$ higher than that of the best p-type BAS sample at the same temperature.

In simple metals and degenerate semiconductors the thermopower depends on the charge carrier concentration as $S \propto$ $n^{-2 / 3}$. In Fig. 3 we show such a plot for the room-temperature thermopower of Ce-BAS and La-BAS, together with data for $\mathrm{p}$ and n-type BAS samples from the literature ${ }^{20}$. Whereas La-BAS nicely fits into the series, Ce-BAS has a strongly enhanced thermopower: $|S|$ is by as much as $50 \%$ enhanced with respect to the value expected from its carrier concentration. May this again be attributed to the Kondo interaction? To answer this question we have performed thermodynamic measurements at low temperatures, which are likely to reveal more insight into the Kondo physics of this system.

The magnetic susceptibility $\chi(T)$ reveals that Ce-BAS is paramagnetic, whereas La-BAS is diamagnetic (Fig. 4a), with no sign of magnetic ordering down to the lowest accessed temperatures. The paramagnetic behaviour of $\mathrm{Ce}-\mathrm{BAS}$ can be attributed to the presence of $\mathrm{Ce}^{+3}$ ions. They have the electron configuration [Xe] $4 f^{1}$ and thus possess one localized, well-shielded electron that carries a magnetic moment. The sixfold degenerate 


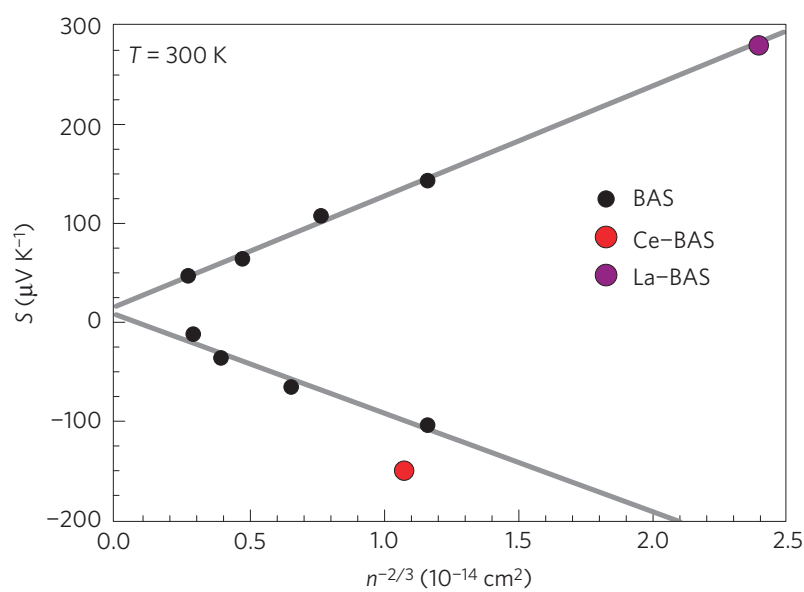

Figure 3 | Thermopower comparison with the rare-earth-free reference material BAS. Room-temperature thermopower of Ce-BAS and La-BAS, together with published values for a series of BAS samples with different Au contents ${ }^{20}$ as a function of the charge carrier concentration, plotted as $n^{-2 / 3}$. The lines are linear fits to the data for BAS.

spin-orbit ground state ${ }^{2} F_{5 / 2}$ is generally split by crystal electric fields. In cubic point symmetry (the situation relevant for the $2 a$ site), a $\Gamma_{7}$ doublet and a $\Gamma_{8}$ quartet result. The temperature dependence of an ensemble of free (non-interacting) moments will
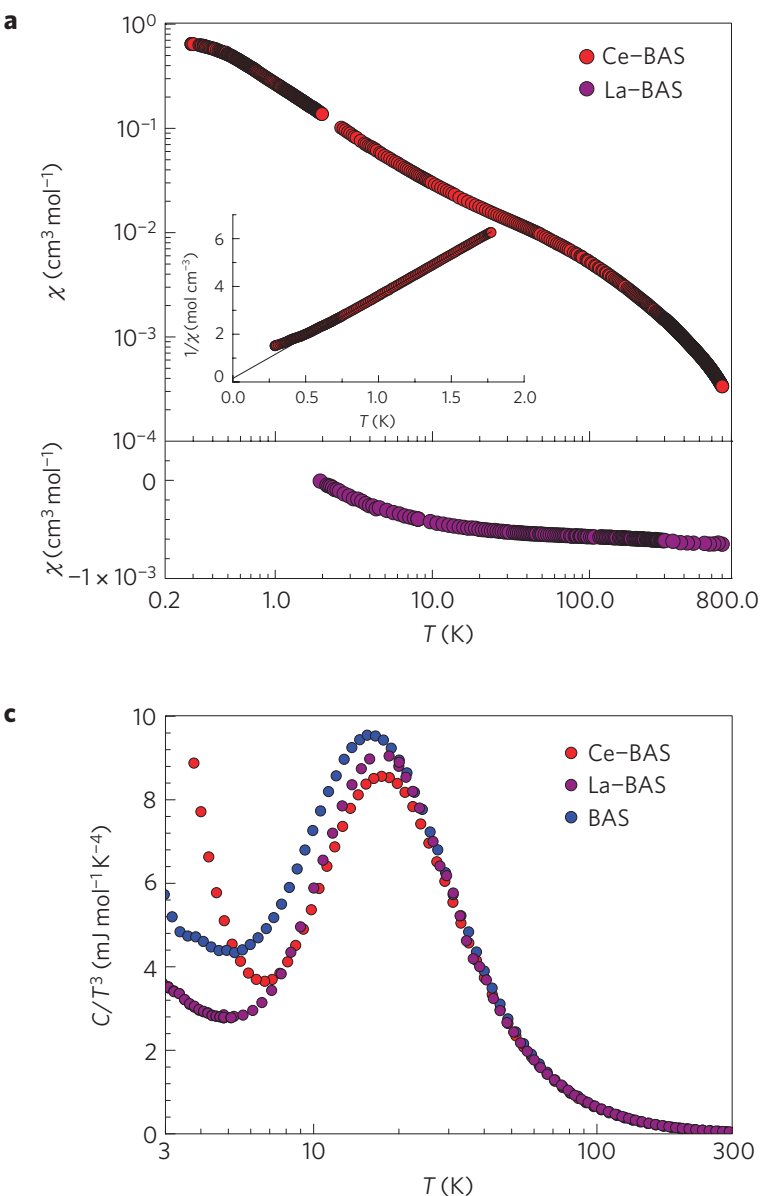

be determined by the thermal population of the different energy levels. As the splitting between the $\Gamma_{7}$ and the $\Gamma_{8}$ state is typically of the order of 50-100 K, the crystal field ground state will govern the lowest temperature properties.

Between about 1 and $6 \mathrm{~K}, \chi(T)$ of Ce-BAS shows Curie-Weisstype behaviour, with an effective moment of $1.48 \mu_{\mathrm{B}}$ per Ce ion and a paramagnetic Weiss temperature close to zero (Fig. 4a, inset). The latter is expected owing to the large distance between nearest-neighbouring $\mathrm{Ce}$ atoms of about $0.9 \mathrm{~nm}$. Below $1 \mathrm{~K}$, the magnetic susceptibility deviates from this Curie-Weiss law. Its tendency to saturate to a constant value can be attributed to the Kondo interaction.

This is further corroborated by the specific heat $C(T)$ of Ce-BAS. It shows a pronounced anomaly in zero magnetic field (Fig. 4b) that can be attributed to the splitting of a Kramers doublet by the Kondo interaction. Both the peak temperature and the peak value increase with applied fields, again behaviour typical for Kondo systems. After subtracting the phonon specific heat of the reference compound La-BAS, the magnetic entropy of the anomaly can be estimated. An entropy of $0.4 R \ln 2$ per mole of $\mathrm{Ce}$, where $R$ is the universal gas constant, is released up to $0.5 \mathrm{~K}$. Twice this temperature is generally considered a good estimate of the Kondo temperature ${ }^{26,27}$. Taking all information together, Ce-BAS behaves as an incoherent Kondo system with a low Kondo temperature of $T_{\mathrm{K}} \approx 1 \mathrm{~K}$ at low temperatures.

This raises an important question: how can this low Kondo temperature lead to an enhancement of the thermopower even
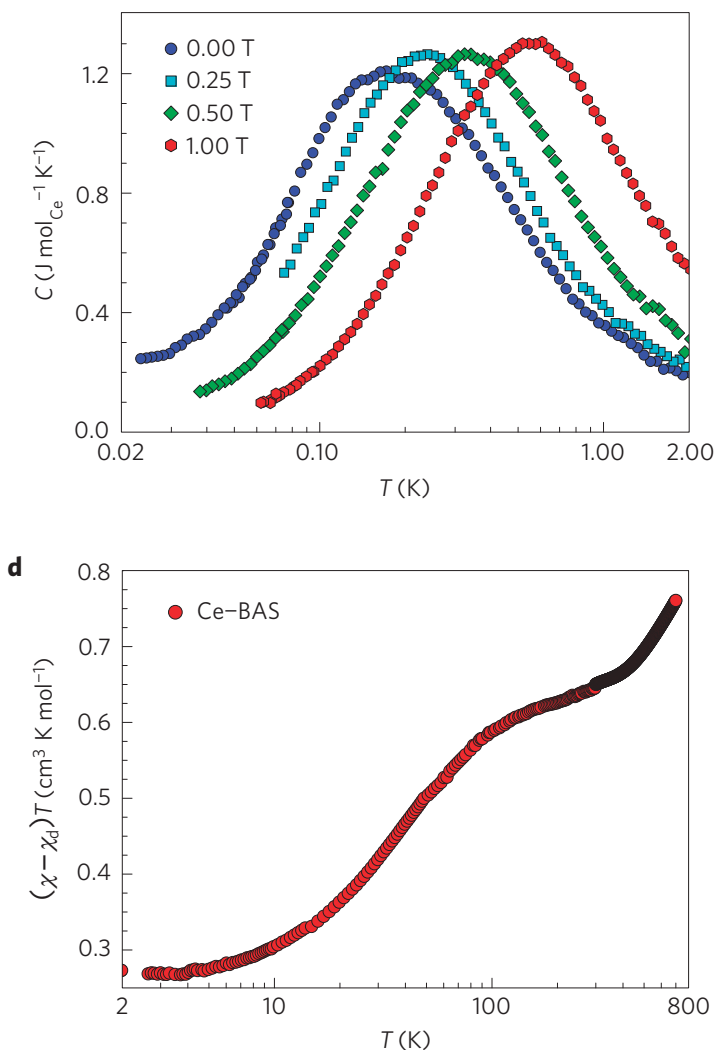

Figure 4 | Thermodynamic properties of the investigated clathrates. a, Magnetic susceptibility of Ce-BAS (top) and La-BAS (bottom) versus temperature. Inset: inverse magnetic susceptibility of Ce-BAS at low temperatures. b, Low-temperature specific heat of Ce-BAS in different magnetic fields. c, Specific heat of Ce-BAS, La-BAS and BAS plotted as $C / T^{3}$ versus temperature. $\mathbf{d}$, Temperature dependence of the paramagnetic contribution to the magnetic susceptibility of $\mathrm{Ce}-\mathrm{BAS}$, plotted as $\chi T$ versus temperature. 
above room temperature? We argue in the following that a second, much higher Kondo scale emerges in this system as a consequence of spin-phonon coupling.

First we discuss the evidence for rattling in both Ce-BAS and La-BAS. Einstein-like contributions to the specific heat can be nicely revealed by $C / T^{3}$ versus $\ln T$ plots, where they appear as bell-shaped anomalies on top of a background contribution due to a Debye solid plus charge carrier/spin contributions at low temperatures ${ }^{28}$. By the introduction of $\mathrm{Ce}$ and $\mathrm{La}$, the peak observed for BAS is only slightly reduced in amplitude and slightly shifted to higher temperatures (Fig. 4c). This can be attributed to a slightly reduced amount of free space ${ }^{29}$ for the $\mathrm{Ba}$ ions in the small cages, the diameter of which have shrunk by $0.81 \%(0.89 \%)$ by the introduction of the smaller ion $\mathrm{Ce}(\mathrm{La})$ (Supplementary Information). Surprisingly, the atomic displacement parameter at the $2 a$ site shows a relative enhancement in Ce-BAS (La-BAS) compared with values for BAS (Supplementary Information). This indicates that the rattling amplitude of the $\mathrm{Ce}(\mathrm{La})$ ions in the small cages is enhanced compared with that of Ba at the same site in BAS. Thus, rattling is a characteristic of Ce-BAS (La-BAS) just as it is for rare-earth-free clathrates. In the following we argue that this has profound implications on the Kondo interaction.

One of the simplest models that treats the interplay between localized electrons and local optical phonon modes is the Anderson-Holstein model ${ }^{30}$. It has been shown that, in the regime of strong electron correlation, the Kondo energy scale can be strongly enhanced by the electron-phonon interaction ${ }^{3}$. Rattling in clathrates is a thermally activated process. Thus, local phonon modes are populated only at elevated temperatures. This may lead to a strong renormalization of the Kondo energy scale with increasing temperature. We argue below that the temperature dependence of the magnetic susceptibility supports this picture.

The paramagnetic contribution to the magnetic susceptibility of Ce-BAS (Methods) is shown as $\chi T$ versus $T$ in Fig. 4 d. A pure Curie law corresponds to constant behaviour in this plot. We see two regions with weak temperature dependence, one up to about $6 \mathrm{~K}$ (plateau 1) and a second one between about 120 and $400 \mathrm{~K}$ (plateau 2). Qualitatively, the transition between plateau 1 and 2 is most naturally attributed to the thermal population of the upper crystal electric field level. However, the susceptibility reached at plateau 2 is sizably reduced with respect to the local moment limit (free $\mathrm{Ce}^{+3}$ ions would give $\chi T \approx 0.81 \mathrm{~cm}^{3} \mathrm{~K} \mathrm{~mol}^{-1}$ ). Also the increase of the susceptibility above plateau 2 does not fit into a simple crystal electric field level scheme. Instead, the suppression of the susceptibility from the local moment limit, which has already set in at the highest temperature of $700 \mathrm{~K}$ accessed in our experiment, can be attributed to a Kondo temperature of several hundred Kelvin.

Thus, the following picture emerges. The rattling modes of the $\mathrm{Ce}$ ions encapsulated in the cages of the clathrate $\mathrm{Ba}_{6.91} \mathrm{Ce}_{1.06} \mathrm{Au}_{5.56} \mathrm{Si}_{40.47}$ couple to the conduction electrons on the framework, thereby effectively reducing the Coulomb repulsion $U$, and enhancing the system's Kondo temperature by orders of magnitude. This leads to both the early suppression of the magnetic susceptibility from the high-temperature local moment limit and to the $50 \%$ enhancement of the thermopower (and $100 \%$ enhancement of $Z T$ at $600 \mathrm{~K}$ if $\kappa_{1}(600 \mathrm{~K})=\kappa_{1}(480 \mathrm{~K})$ is assumed) over the value of the rare-earth-free reference material. We expect these results to open up a new avenue for research in thermoelectrics. Furthermore, they are likely to stimulate further research into related phenomena, such as magnetically robust $^{31,32}$ and electric dipolar Kondo behaviour ${ }^{33}$, or thermopower enhancements in the negative- $U$ Anderson model ${ }^{34}$.

\section{Methods}

Synthesis, structural and analytical characterization. Polycrystalline starting material was synthesized by melting of high-purity $\mathrm{Ba}, \mathrm{Ce}, \mathrm{Au}$ and $\mathrm{Si}$ in a cold copper boat using high-frequency heating. The resulting samples were polyphased, with the clathrate-I phase being the majority phase. Long-term annealing did not change the phase relations. The amount of Ce detected by EDX in the clathrate phase was, with about 1.2 at.\% (0.65 Ce per formula unit), smaller than in the starting material.

Phase-pure Ce-containing clathrates (Ce-BAS) were finally obtained by off-stoichiometric crystal growth by the floating-zone melting technique using optical heating in a four-mirror furnace (Crystal Corporation). The obtained clathrate sample of about $5 \times 4 \times 3 \mathrm{~mm}^{3}$ consisted of a few single crystals and was phase pure according to powder XRD (Siemens D5000 diffractometer, Fig. 1b) and scanning electron microscopy (Philips XL30 ESEM, Fig. 1c). High-resolution transmission electron microscopy (FEI TECNAI F20) proved the absence of nano-inclusions (Fig. 1d). The La-containing $\mathrm{Ba}-\mathrm{Au}-\mathrm{Si}$ clathrate (La-BAS) was prepared with a similar technique.

Rietveld refinements of the powder XRD patterns of powdered single-crystalline samples yield the lattice parameters $a=1.0395(2) \mathrm{nm}$ and $a=1.0392(2) \mathrm{nm}$ for Ce-BAS and La-BAS, respectively. These are sizably smaller than published values for $\mathrm{Ba}_{8} \mathrm{Au}_{x} \mathrm{Si}_{40-x}$ clathrates with a similar Au content ${ }^{20,25,35}$. As the ionic radii of $\mathrm{Ce}$ and $\mathrm{La}$ are smaller than the one of $\mathrm{Ba}$ this supports the incorporation of the rare earth atoms in the clathrate phase. Furthermore, EDX and WDX analyses (EDX: EDAX New XL-30 135-10 UTW+ detector, WDX: Microspec WDX-600), with a single-phase $\mathrm{Ba}_{8} \mathrm{Au}_{5} \mathrm{Si}_{41}$ sample as the standard, confirm the presence of $\mathrm{Ce}$ and $\mathrm{La}$ in the crystals. The average crystal compositions measured by EDX are $\mathrm{Ba}_{6.91 \pm 0.17} \mathrm{Ce}_{1.06 \pm 0.12} \mathrm{Au}_{5.56 \pm 0.25} \mathrm{Si}_{40.47 \pm 0.43}$ and $\mathrm{Ba}_{6.99 \pm 0.17} \mathrm{La}_{1.23 \pm 0.12} \mathrm{Au}_{5.91 \pm 0.25} \mathrm{Si}_{39.87 \pm 0.43}$. Single-crystal XRD investigations are reported in Supplementary Information.

Physical property measurements. The magnetic properties below room temperature were measured in a superconducting quantum interference device magnetometer (Cryogenics S700X) after cooling down the samples in zero applied field. Above room temperature, the susceptibility was measured by the vibrating sample magnetometer option of a Physical Property Measurement System (PPMS, Quantum Design). The specific heat below $2 \mathrm{~K}$ was measured with a relaxation-type method using a home-built set-up in a ${ }^{3} \mathrm{He} /{ }^{4} \mathrm{He}$ dilution refrigerator. Above $2 \mathrm{~K}$, it was measured by the same technique in a PPMS. Electrical resistivity and Hall effect measurements were done by standard 4-point and 6-point techniques, with alternating d.c. current in a PPMS. Below room temperature, thermopower was determined by a two-heater method in a home-built cryostat. Above $300 \mathrm{~K}$, thermopower was measured simultaneously with the electrical resistivity using a standard 4-point steady-state d.c. technique (ZEM 3, Ulvac-Riko). The thermal conductivity was determined from thermal diffusivity measurements using a standard laser-flash experiment (Anter, Flashline 3000FT - S2) and measured specific heat data.

Diamagnetic susceptibility contributions. To correct the magnetic susceptibility of Ce-BAS for diamagnetic contributions we use the $4 f$-free reference material $\mathrm{La}-\mathrm{BAS}$, which is expected to have very similar diamagnetic contributions. Its susceptibility has the form

$$
\chi=\chi_{\mathrm{d}, \mathrm{cs}}+\chi_{\mathrm{d}, \text { ring }}+\chi_{\mathrm{p}, \text { imp }}+\frac{2}{3} \chi_{\mathrm{p}, \text { Pauli }}
$$

where $\chi_{\mathrm{d}, \mathrm{cs}}$ and $\chi_{\mathrm{d} \text {,ring }}$ are diamagnetic contributions of closed shells and due to ring currents ${ }^{35}$, respectively. $\chi_{\mathrm{p}, \text { imp }}$ is a paramagnetic contribution due to a small amount of magnetic impurities; it is relevant only at the lowest temperatures. $\chi_{\mathrm{p}, \text { Pauli }}$ is the Pauli paramagnetic contribution of the conduction electrons. The factor $2 / 3$ accounts for Landau diamagnetism. We determine $\chi_{\mathrm{p}, \text { Pauli }}$ and $\chi_{\mathrm{p} \text {,imp }}$ of La-BAS from Hall effect measurements and a fit to a Curie-Weiss law at low temperature, respectively, and then subtract both of these terms from the total measured $\chi(T)$ data of La-BAS. The result is identified as the total diamagnetic contribution $\chi_{\mathrm{d}}$ of both La-BAS and Ce-BAS.

Received 19 April 2013; accepted 15 August 2013; published online 22 September 2013

\section{References}

1. Toberer, E. S., Christensen, M., Iversen, B. B. \& Snyder, G. J. High temperature thermoelectric efficiency in $\mathrm{Ba}_{8} \mathrm{Ga}_{16} \mathrm{Ge}_{30}$. Phys. Rev. B 77, 075203 (2008).

2. Paschen, S. in Thermoelectrics Handbook (ed. Rowe, D. M.) Ch. 15 (CRC, 2006).

3. Hotta, T. Enhanced Kondo effect in an electron system dynamically coupled with local optical phonons. J. Phys. Soc. Jpn 76, 084702 (2007).

4. Christensen, M. et al. Avoided crossing of rattler modes in thermoelectric materials. Nature Mater. 7, 811-815 (2008).

5. Euchner, H. et al. Phononic filter effect of rattling phonons in the thermoelectric clathrate $\mathrm{Ba}_{8} \mathrm{Ge}_{40+x} \mathrm{Ni}_{6-x}$. Phys. Rev. B 86, 224303 (2012).

6. Slack, G. A. in CRC Handbook of Thermoelectrics (ed. Rowe, D. M.) Ch. 34 (CRC, 1995).

7. Saramat, A. et al. Large thermoelectric figure of merit at high temperature in Czochralski-grown clathrate $\mathrm{Ba}_{8} \mathrm{Ga}_{16} \mathrm{Ge}_{30}$. J. Appl. Phys. 99, 023708 (2006). 
8. Anno, H., Hokazono, M., Kawamura, M., Nagao, J. \& Matsubara, K. Thermoelectric properties of $\mathrm{Ba}_{8} \mathrm{Ga}_{x} \mathrm{Ge}_{46-x}$ clathrate compounds. Proc. 21st Int. Conf. on Thermoelectrics 77-80 (Long Beach, 2002).

9. Rowe, D. M., Kuznetsov, V. L., Kuznetsova, L. A. \& Min, G. Electrical and thermal transport properties of intermediate-valence $\mathrm{YbAl}_{3}$. J. Phys. D 35, 2183-2186 (2002).

10. Sales, B. C. et al. Magnetic, transport, and structural properties of $\mathrm{Fe}_{1-x} \mathrm{Ir}_{x} \mathrm{Si}$. Phys. Rev. B 50, 8207-8213 (1994).

11. Jie, Q. et al. Electronic thermoelectric power factor and metal-insulator transition in $\mathrm{FeSb}_{2}$. Phys. Rev. B 86, 115121 (2012).

12. Zlatić, V. \& Monnier, R. Theory of the thermoelectricity of intermetallic compounds with Ce or Yb ions. Phys. Rev. B 71, 165109 (2005).

13. Coleman, P. in Fundamentals and Theory Vol. 1 (eds Kronmüller, H. \& Parkin, S.) 95-148 (Handbook of Magnetism and Advanced Magnetic Materials, Wiley, 2007).

14. Kawaguchi, T., Tanigaki, K. \& Yasukawa, M. Silicon clathrate with an $f$-electron system. Phys. Rev. Lett. 85, 3189-3192 (2000).

15. Pacheco, V., Carrillo-Cabrera, W., Tran, V. H., Paschen, S. \& Grin, Y. Comment on "silicon clathrate with an $f$-electron system". Phys. Rev. Lett. 87, 099601 (2001).

16. Kawaguchi, T., Tanigaki, K. \& Yasukawa, M. Comment on "silicon clathrate with an $f$-electron system": Reply. Phys. Rev. Lett. 87, 099602 (2001).

17. Tang, X., Li, P., Deng, S. \& Zhang, Q. High temperature thermoelectric transport properties of double-atom-filled clathrate compounds $\mathrm{Yb}_{x} \mathrm{Ba}_{8-x} \mathrm{Ga}_{16} \mathrm{Ge}_{30}$. J. Appl. Phys. 104, 013706 (2008).

18. Kovnir, K. et al. Introducing a magnetic guest to a tetrel-free clathrate: Synthesis, structure, and properties of $\mathrm{Eu}_{x} \mathrm{Ba}_{8-x} \mathrm{Cu}_{16} \mathrm{P}_{30}(0 \leq x \leq 1.5)$. Inorg. Chem. 50, 10387-10396 (2011).

19. Schäfer, H. On the problem of polar intermetallic compounds: The stimulation of E. Zintl's work for the modern chemistry of intermetallics. Ann. Rev. Mater. Sci. 15, 1-42 (1985).

20. Aydemir, U. et al. Low-temperature thermoelectric, galvanomagnetic and thermodynamic properties of the type-I clatharate $\mathrm{Ba}_{8} \mathrm{Au}_{x} \mathrm{Si}_{46-x}$. Phys. Rev. $B$ 84, 195137 (2011).

21. Fert, A. \& Levy, P. M. Theory of the Hall effect in heavy-fermion compounds. Phys. Rev. B 36, 1907-1916 (1987).

22. Nguyen, L. T. K. et al. Atomic ordering and thermoelectric properties of the n-type clathrate $\mathrm{Ba}_{8} \mathrm{Ni}_{3.5} \mathrm{Ge}_{42.1} \square_{0.4}$. Dalton Trans. 39, 1071-1077 (2010).

23. Nakatsuji, S. et al. Intersite coupling effects in a Kondo lattice. Phys. Rev. Lett. 89, 106402 (2002).

24. Candolfi, C. et al. High temperature thermoelectric properties of the type-I clathrate $\mathrm{Ba}_{8} \mathrm{Au}_{x} \mathrm{Si}_{46-x}$. J. Appl. Phys. 111, 043706 (2012).

25. Zeiringer, I. et al. The ternary system Au-Ba-Si: Clathrate solution, electronic structure, physical properties, phase eqilibria and crystal structures. Acta Mater. 60, 2324-2336 (2012).
26. Mel'nikov, V. I. Thermodynamics of the Kondo problem. JETP Lett. 35, 511-515 (1982)

27. Gegenwart, P., Si, Q. \& Steglich, F. Quantum criticality in heavy-fermion metals. Nature Phys. 4, 186-197 (2008).

28. Junod, A., Jarlborg, T. \& Muller, J. Heat-capacity analysis of a large number of A15-type compounds. Phys. Rev. B 27, 1568-1585 (1983).

29. Suekuni, K., Avila, M. A., Umeo, K. \& Takabatake, T. Cage-size control of guest vibration and thermal conductivity in $\mathrm{Sr}_{8} \mathrm{Ga}_{16} \mathrm{Si}_{30-x} \mathrm{Ge}_{x}$. Phys. Rev. B 75, 195210 (2007).

30. Hewson, A. C. \& Meyer, D. Numerical renormalization group study of the Anderson-Holstein impurity model. J. Phys. Condens. Matter 14, 427-445 (2002).

31. Sanada, S. et al. Exotic heavy-fermion state in filled skutterudite $\mathrm{SmOs}_{4} \mathrm{Sb}_{12}$. J. Phys. Soc. Jpn 74, 246-249 (2005).

32. Costi, T. A. \& Zlatić, V. Charge Kondo anomalies in PbTe doped with $\mathrm{Tl}$ impurities. Phys. Rev. Lett. 108, 036402 (2012).

33. Hotta, T. \& Ueda, K. Electric dipolar Kondo effect emerging from a vibrating magnetic ion. Phys. Rev. Lett. 108, 247214 (2012).

34. Andergassen, S., Costi, T. A. \& Zlatić, V. Mechanism for large thermoelectric power in molecular quantum dots described by the negative-U Anderson model. Phys. Rev. B 84, 241107(R) (2011)

35. Herrmann, R. F. W., Tanigaki, K., Kawaguchi, T., Kuroshima, S. \& Zhou, O Electronic structure of Si and Ge gold-doped clathrates. Phys. Rev. B 60, 13245-13248 (1999).

\section{Acknowledgements}

We acknowledge financial support from the Austrian Science Fund (projects P19458-N16, TRP 176-N22 and I623-N16) and the European Research Council (Advanced Grant QuantumPuzzle, no. 227378), and help from T. Pippinger and R. Miletich-Pawliczek (Vienna University) during single-crystal XRD measurements on La-BAS. TEM images were made by J. Bernardi and M. Stöger-Pollach at USTEM (Vienna University of Technology).

\section{Author contributions}

A.P. and S.P. designed the research. A.P. synthesized the material; A.S., M.I., R.S. M.W., H.W., K.N. and K.H. performed the measurements. A.P., A.S., H.W., K.H. and S.P. analysed the data. A.P., A.S. and S.P. prepared the manuscript.

\section{Additional information}

Supplementary information is available in the online version of the paper. Reprints and permissions information is available online at www.nature.com/reprints. Correspondence and requests for materials should be addressed to A.P. or S.P.

\section{Competing financial interests}

The authors declare no competing financial interests. 